\title{
Therapeutic potential of EGCG on acute renal damage in a rat model of obstructive nephropathy
}

\author{
PING ZHOU ${ }^{1,2 *}$, JIN FENG YU ${ }^{3 *}$, CHENG GUANG ZHAO ${ }^{1}$, FENG XUAN SUI ${ }^{3}, \mathrm{XU} \mathrm{TENG}^{1}$ and $\mathrm{YU} \mathrm{BIN} \mathrm{WU}^{1}$ \\ ${ }^{1}$ Department of Pediatrics, Shengjing Hospital Of China Medical University, Shenyang 110004; \\ ${ }^{2}$ Department of Pediatrics, The Second Affiliated Hospital of Harbin Medical University, Harbin 150086; \\ ${ }^{3}$ Department of Pediatrics, The Hong Qi Hospital of Mudanjiang Medical University, Mudanjiang 157011, P.R. China
}

Received July 14, 2012; Accepted September 28, 2012

DOI: $10.3892 / \mathrm{mmr} .2013 .1296$

\begin{abstract}
As a major active component in green tea, (-)-epigallocatechin 3-O-gallate (EGCG) has many anti-oxidative activities. This study investigated whether intraperitoneal administration of EGCG was capable of suppressing oxidative stress in rats with unilateral ureteral obstruction (UUO) and probed the potential mechanisms involved. In total, 150 adult male rats were randomly divided into 5 groups $(n=30$ each): the control group (group N); the unilateral ureteral obstruction (UUO) group (group C), where the unilateral ureter was ligated resulting in an obstructive nephropathy model; and the EGCG group (group T), following unilateral ureteral ligation, rats were intraperitoneally injected with EGCG at a dosage of $2.5\left(\mathrm{~T}_{1}\right), 5\left(\mathrm{~T}_{2}\right)$ and $10 \mathrm{mg} / \mathrm{kg} /$ day $\left(\mathrm{T}_{3}\right)$. Each group of rats was sacrificed $72 \mathrm{~h}$ after surgery. We evaluated the effects of EGCG on the reactive oxygen species (ROS), reduced glutathione (GSH), oxidized glutathione (GSSG) and glutathione in the renal tissue of rats. Immunohistochemistry and western blot analysis were applied to detect nuclear factor erythoid-derived 2 -related factor 2 (Nrf2) and $\gamma$-glutamylcysteine synthetase $(\gamma$-GCS) protein expression. Real-time PCR was performed to detect the mRNA levels of Nrf 2 and $\gamma$-GCS. Changes in renal ultrastructure were also observed using electron microscopy. There was no significant difference in GSH, and compared with group N, ROS, GSSG and total GSH levels were much higher in the $\mathrm{T}$ groups $(\mathrm{p}<0.01)$, while much lower than those of group $C(p<0.01)$. Protein levels of Nrf2 and $\gamma-G C S$ and the mRNA levels of Nrf 2 and $\gamma$-GCS notably increased in EGCGtreated rats (all $\mathrm{p}<0.05)$. Furthermore, electron microscopy showed that renal ultrastructure was improved in the treatment
\end{abstract}

Correspondence to: Professor $\mathrm{Yu}$ Bin Wu, Department of Pediatrics, Shengjing Hospital of China Medical, No. 36, Sanhao Street, Heping Street, Shenyang 110004, P.R. China

E-mail: yubinwucn@163.com

*Contributed equally

Key words: obstructive nephropathy, oxidative stress, Nrf2, $\gamma$-GCS, (-)-epigallocatechin 3-O-gallate groups. Our findings suggest that, resulting from suppression of oxidative stress influenced by free radicals, EGCG exerts a protective effect on rats with obstructive nephropathy, and this anti-oxidative effect may be partly induced by activating the Nrf2 signaling pathway.

\section{Introduction}

Obstructive uropathy refers to the presence of structural or functional changes in the urinary tract that impede the normal flow of urine. Hydronephrosis denotes dilation of the urinary tract. Obstructive nephropathy is a relatively common entity that is treatable and often reversible. It occurs at all ages, from infants to elderly subjects. It is the result of functional or anatomic lesions located in the urinary tract. Obstructive nephropathy is one of the most serious diseases in children and has been the most common cause of end-stage renal failure (1). The kidney is important in blood filtration, concentration, excretion of waste products and electrolytes, and reabsorption of nutrients, thus serving as a main organ for maintaining homeostatic conditions (2). Active transport of these metabolites through the renal tubules increases the opportunities for tubule cells to produce or come in contact with harmful agents, such as reactive oxygen species (ROS), indicating that the kidney is at high risk of oxidative stress due to its metabolic action. Oxidative stress refers to a disrupted redox equilibrium between the production of free radicals and the ability of cells to protect against damage caused by these molecules. Oxidative stress has been implicated as a major cause of tissue injuries in a variety of human diseases, including obstructive nephropathy. Free radicals specifically play important roles in the oxidative stress pathway (3).

The medicinal benefits of drinking green tea have been known in Asian countries since ancient times. The consumption of green tea has attracted much recent attention due to its emerging beneficial health effects. Green tea is one of the most popular and widely consumed beverages and is rich in a variety of antioxidant catechin polyphenols (4-6). The major polyphenols present in green tea are (-)-epicatechin, (-)-epicatechin-3-gallate, (-)-epigallocatechin and (-)-epigallocatechin-3-gallate (EGCG), which comprise $30-42 \%$ of solid green tea extract (6). Among these components, EGCG is the most abundant and most active catechin derivative, with potent 
antioxidant and chemopreventive activities (7). Since acute obstructive nephropathy is associated with increased oxidative stress in the tissue, it is likely that the protective effects of catechins are mainly due to their anti-oxidative properties. Catechins are known as effective scavengers of ROS, which are also involved in modulation of gene expression (8). Recently, much attention has been focused on EGCG, which has been reported to possess anti-oxidative, anti-inflammatory and anti-carcinogenic effects $(4,9,10)$.

Therefore, in order to ascertain whether oxidative stress was involved in the mechanism of obstructive nephropathy in rats and to evaluate the effect of EGCG as a representative polyphenol, we investigated the effect of EGCG on oxidative stress in UUO rats with obstructive nephropathy and probed the underlying mechanisms. The chemical structure of EGCG is illustrated in Fig. 1.

\section{Materials and methods}

Animals. Male, Sprague Dawley (SD) rats (120-150 g, 6-8 weeks old; China Medical University Experimental Animal Research Center, Shenyang, China) were used in these experiments. The rats were housed in standard cages (four animals per cage) and fed standard laboratory chow and tap water ad libitum. A constant photoperiod (14 h light and $10 \mathrm{~h}$ dark) and constant temperature of $20^{\circ} \mathrm{C}$ were maintained. Procedures for care and handling of animals used in this study were approved by the ethics committee of China Medical University and carried out in accordance with guidelines established by the China Medical University Experimental Animal Research Centre.

Experimental design. In total, 150 adult male rats were randomly divided into 5 groups ( $\mathrm{n}=30$ each): control group (group $\mathrm{N}$ ), a normal group of rats that underwent sham surgery; unilateral ureteral obstruction (UUO) group (group C), where the unilateral ureter was ligated resulting in an obstructive nephropathy model; EGCG group (group T), where rats were intraperitoneally injected with the EGCG at dosage of $2.5 \mathrm{mg} / \mathrm{kg}\left(\mathrm{T}_{1}\right), 5 \mathrm{mg} / \mathrm{kg}\left(\mathrm{T}_{2}\right)$ and $10 \mathrm{mg} / \mathrm{kg}\left(\mathrm{T}_{3}\right) /$ day (each dose, $n=30$ ), following unilateral ureteral ligation. When the 72-h experiment was performed, the normal and control groups received physiological saline.

Animal surgery and sampling. Briefly, rats were anesthetized, laparotomy was performed, and the left ureter identified and ligated at two points along the ureter $1 \mathrm{~cm}$ apart with 4/0 silk, 3-5 mm below the renal hilum. The sham-surgery was performed in the same way; the rats of the normal group (ten rats) were also laparatomized, the ureter exposed, but no ligature was made. Following closure of the abdomen, the animals were returned to the cage with free access to standard lab chow and water ad libitum. Rats were allowed to recover for $72 \mathrm{~h}$ prior to tissue collection. Unilateral obstructive nephropathy was confirmed by sacrificing all the rats $72 \mathrm{~h}$ after surgery. The obstructed kidneys were then fixed for 4 $\mathrm{h}$ in $4 \%$ formalin and embedded in paraffin for immunohistochemistry analysis. The remaining portions of each sample were diced finely in the presence of liquid nitrogen, and stored until analysis.

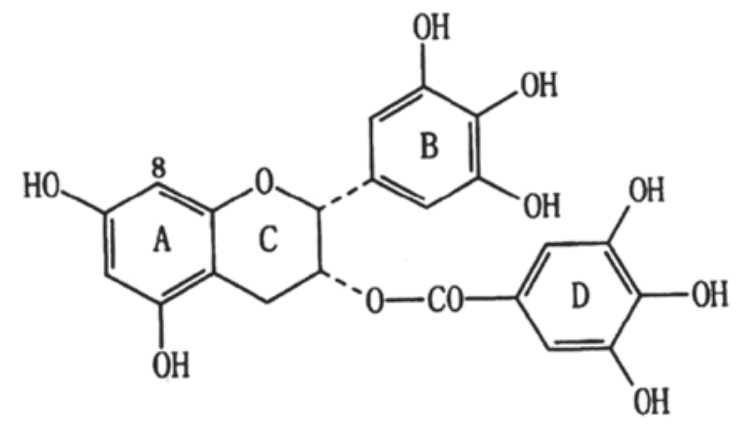

Figure 1. Chemical structure of EGCG.

Measurement of ROS, GSH, GSSG, total GSH and GSH/ GSSG. Briefly, tissue was homogenized in TES/SB buffer consisting of $20 \mathrm{mM}$ Tris, $1 \mathrm{mM}$ EDTA, $250 \mathrm{mM}$ sucrose, $20 \mathrm{mM}$ sodium borate and $2 \mathrm{mM}$ serine. The homogenates were centrifuged at $4^{\circ} \mathrm{C}$ for $10 \mathrm{~min}$ at $10,000 \mathrm{x} \mathrm{g}$, and the supernatants were obtained. The content of ROS, GSH, GSSG and total glutathione was measured by commercial kits (Nanjing Jiancheng Bioengineering Institute) according to the manufacturer's instructions.

Immunohistochemistry. Sections (5 $\mu \mathrm{m}$ thickness) were prepared and subjected to the immunoperoxidase method. Endogenous peroxidase was eliminated by treatment with $3 \% \mathrm{H}_{2} \mathrm{O}_{2} / 10 \%$ methanol phosphate buffered saline (PBS) for $20 \mathrm{~min}$ at room temperature. After washing with water and 0.05 M PBS ( $\mathrm{pH} 7.4$ ), slides were blocked with $5 \%$ bovine serum albumin (BSA) in PBS for $20 \mathrm{~min}$ at room temperature to prevent non-specific protein binding. The slides were then incubated with 1:100 diluted specific primary antibody for Nrf2 and $\gamma$-GCS (Santa Cruz Biotechnology, Santa Cruz, $\mathrm{CA}, \mathrm{USA}$ ) in PBS containing $5 \% \mathrm{BSA}$ overnight at $4^{\circ} \mathrm{C}$. The sections were rinsed in $0.05 \mathrm{M}$ PBS containing $0.1 \% \mathrm{BSA}$. After washing with PBS, the slides were incubated with a biotinylated goat peroxidase conjugated secondary antibody and $0.1 \%$ DAB substrate, using the standard streptavidin-biotin-based method. For the negative control, slides were processed without primary antibody. The optical densities of Nrf2 and $\gamma$-GCS bands from the membranes were determined by densitometric scanning using a Nikon Eclipse Scanjet E800 photo scanner and MoticMed System 6.0 software. The value of the optical density of each protein band was expressed as the mean \pm standard deviation.

Nrf2 and $\gamma$-GCS gene expression. When rats were sacrificed, renal tissue was immediately frozen in liquid nitrogen for RNA extraction. Total RNA was isolated from rat renal tissues with TRIzol reagent (Invitrogen, Carlsbad, CA, USA). Total RNA was reverse-transcribed using the PrimeScript ${ }^{\mathrm{TM}}$ RT Reagent kit (Takara Biotechnology Dalian, China) according to the manufacturer's instructions. Quantitative real-time PCR was performed for Nrf2, $\gamma$-GCS and GAPDH using the following primers pairs: Nrf2, sense 5'-TTGATTGACATCC TTTGG-3' and antisense 5'-GTTCCTTCTGGAGTTGCT-3'; $\gamma$-GCS, sense 5'-ATCTACCACGCAGTCAAG-3' and antisense 5'-CCGCCATTCAGTAACAAC-3'; GAPDH, sense 
Table I. GSH, GSSG, GSH/GSSG, total glutathione and ROS in renal tissue (means \pm SEM, n=10).

\begin{tabular}{lccccc}
\hline & & & \multicolumn{3}{c}{ EGCG } \\
\cline { 4 - 6 } & $\mathrm{N}$ & $\mathrm{C}$ & $\mathrm{T}_{1}$ & $\mathrm{~T}_{2}$ & $\mathrm{~T}_{3}$ \\
\hline GSH (mg/mg pro) & $1.32 \pm 0.29$ & $1.31 \pm 0.11$ & $1.34 \pm 0.28$ & $1.33 \pm 0.52$ & $1.34 \pm 0.47$ \\
GSSG $(\mu$ mol/l) & $0.08 \pm 0.04$ & $0.73 \pm 0.03^{\mathrm{a}}$ & $0.71 \pm 0.05^{\mathrm{a}, \mathrm{b}}$ & $0.57 \pm 0.03^{\mathrm{a}, \mathrm{c}}$ & $0.55 \pm 0.04^{\mathrm{a}, \mathrm{c}}$ \\
Total glutathione $(\mu \mathrm{mol} / \mathrm{l})$ & $0.59 \pm 0.04$ & $1.47 \pm 0.08^{\mathrm{a}}$ & $1.46 \pm 0.06^{\mathrm{a}, \mathrm{b}}$ & $1.24 \pm 0.06^{\mathrm{a}, \mathrm{c}}$ & $1.21 \pm 0.05^{\mathrm{a}, \mathrm{c}}$ \\
GSH/GSSG & $5.25 \pm 0.07$ & $0.59 \pm 0.09^{\mathrm{a}}$ & $0.60 \pm 0.07^{\mathrm{a}, \mathrm{b}}$ & $0.75 \pm 0.08^{\mathrm{a}, \mathrm{c}}$ & $0.78 \pm 0.09^{\mathrm{a}, \mathrm{c}}$ \\
ROS (U/mg pro) & $1617 \pm 124$ & $3782 \pm 207^{\mathrm{a}}$ & $3779 \pm 183^{\mathrm{a}, \mathrm{b}}$ & $3273 \pm 176^{\mathrm{a}, \mathrm{c}}$ & $3114 \pm 191^{\mathrm{a}, \mathrm{c}}$ \\
\hline
\end{tabular}

${ }^{a} \mathrm{p}<0.01$ versus normal values. ${ }^{b} \mathrm{p}<0.05$ versus control values. ${ }^{\mathrm{c}} \mathrm{p}<0.01$ versus control values. ROS, reactive oxygen species; GSH, reduced glutathione; GSSG, oxidized glutathione; EGCG, (-)-epigallocatechin 3-O-gallate.

5'-TGTGTCCGTCGTGGATCTGA-3' and antisense 5'-ATGGTGGTGAAGACGCCAGTA-3'. Real time-PCR analysis was performed using an $\mathrm{ABI} 7500$ with the following thermal cycling conditions: 1 cycle at $95^{\circ} \mathrm{C}$ for $10 \mathrm{sec}$, followed by 40 cycles at $95^{\circ} \mathrm{C}$ for $5 \mathrm{sec}$ and $60^{\circ} \mathrm{C}$ for $34 \mathrm{sec}$. All samples were run in triplicate. The amplification results were detected and analyzed using the SDS real-time PCR detection system. The gene signals were standardized against the corresponding GAPDH signal, and results were expressed as the ratio of each molecule to GAPDH.

Western blot analysis. The frozen renal tissue was immediately homogenized, the protein was solubilized in radioimmunoprecipitation buffer, and the total protein was separated on a $12 \%$ acrylamide SDS-PAGE gel and electroblotted to a nitrocellulose membrane. The membrane was blocked with $5 \%(w / v)$ fat-free milk and then incubated with a monoclonal antibody to Nrf2 and $\gamma$-GCS (rat origin, 1:1000) (Santa Cruz Biotechnology) overnight at $4^{\circ} \mathrm{C}$, followed by incubation with horseradish peroxidase-conjugated secondary antibodies (1:1000) for $1 \mathrm{~h}$ at room temperature. Membranes were exposed to chemiluminescent reagents and then to X-ray film. The average intensity of the bands was determined using Tanon Image software. Data were collected in terms of average intensity of bands of Nrf2 and $\gamma$-GCS per average intensity of bands of $\beta$-actin, and imported to a spreadsheet (Excel; Microsoft, USA).

Electromicroscope. From the unligated right and from the ligated left kidney, large tissue blocks extending from the renal capsule to at least the upper third of the inner zone were immersed for at least $24 \mathrm{~h}$ in the fixative solution, to which $1 \%$ glutardialdehyde was added. This tissue was then embedded into epoxy resin and used for electron microscopy. Ultrathin $(80 \mathrm{~nm})$ sections were cut with an ultramicrotome. Ultrathin sections were postfixed with osmium tetroxyde and contrasted with uranyl acetate and studied with a CM100 Philips electron microscope.

Statistical analysis. All data were presented as the means \pm SEM. One-way analysis of variance and independent t-test were used for statistical analysis of the differences between the groups. $\mathrm{P}<0.05$ was considered to indicate a statistically significant difference.

\section{Results}

Measurement of ROS, GSH, GSSG, total GSH and GSSG. Compared with group N, the ROS and GSSG levels of kidneys in group $\mathrm{C}$ were much higher, and the ratio of GSH/GSSG was much lower. There was no significant difference in GSH compared with group N. ROS, GSSG and total GSH level were much higher in the $\mathrm{T}$ groups $(\mathrm{p}<0.01)$, while much lower than those of group $\mathrm{C}(\mathrm{p}<0.01$; Table I).

Immunohistochemistry. The expression of Nrf2 protein was mainly focused on the renal tubular epithelial cells of rat kidney cortex and medulla. Microscopic observation showed stained nuclei of the renal tubular epithelial cells and dark brown particles. In the immunohistochemical analysis of Nrf2 expression, higher average optical density was found in group $\mathrm{C}$ and the treatment groups compared with group $\mathrm{N}(\mathrm{p}<0.01)$. Compared with group $C$, higher average optical density in the $T_{1}$ group was observed $(\mathrm{p}<0.05)$, and even higher average optical density in the $\mathrm{T}_{2}$ and $\mathrm{T}_{3}$ groups was found ( $<<0.01 ;$ Fig. 2).

The expression of $\gamma$-GCS protein was mainly focused on the renal tubular epithelial cells of the cortex and medulla in rat kidneys. Microscopic observation showed staining in the cytoplasm of the renal tubular epithelial cells and dark brown particles. In the immunohistochemical analysis of $\gamma$-GCS expression, higher average optical density was found in group $\mathrm{C}$ and the treatment groups compared with group $\mathrm{N}(\mathrm{p}<0.01)$. Compared with group $C$, higher average optical density in the $\mathrm{T}_{1}$ group was observed $(\mathrm{p}<0.05)$, and even higher average optical density in the $T_{2}$ and $T_{3}$ groups was found ( $<<0.01 ;$ Fig. 3 ).

Nrf2 and $\gamma$-GCS mRNA expression. Compared with group $\mathrm{N}$, Nrf2 and $\gamma$-GCS mRNA relative copy number in group $C$ and each treatment group were significantly enhanced $(p<0.01)$. Compared with C group, Nrf2 and $\gamma$-GCS mRNA relative copy number in the $T_{1}$ group was enhanced $(p<0.05)$ and significantly enhanced in $\mathrm{T}_{2}$ and $\mathrm{T}_{3}(\mathrm{p}<0.01)$ groups (Fig. 4).

$\gamma$-GCS and Nrf2 protein expression. To elucidate the expression levels of the antioxidant enzyme $\gamma$-GCS, as well as the upstream regulator $\mathrm{Nrf} 2$, we performed western blot analyses of the renal cortex. The results are presented in Fig. 5 . Obstructive nephropathy rats showed increases in the levels of 
A
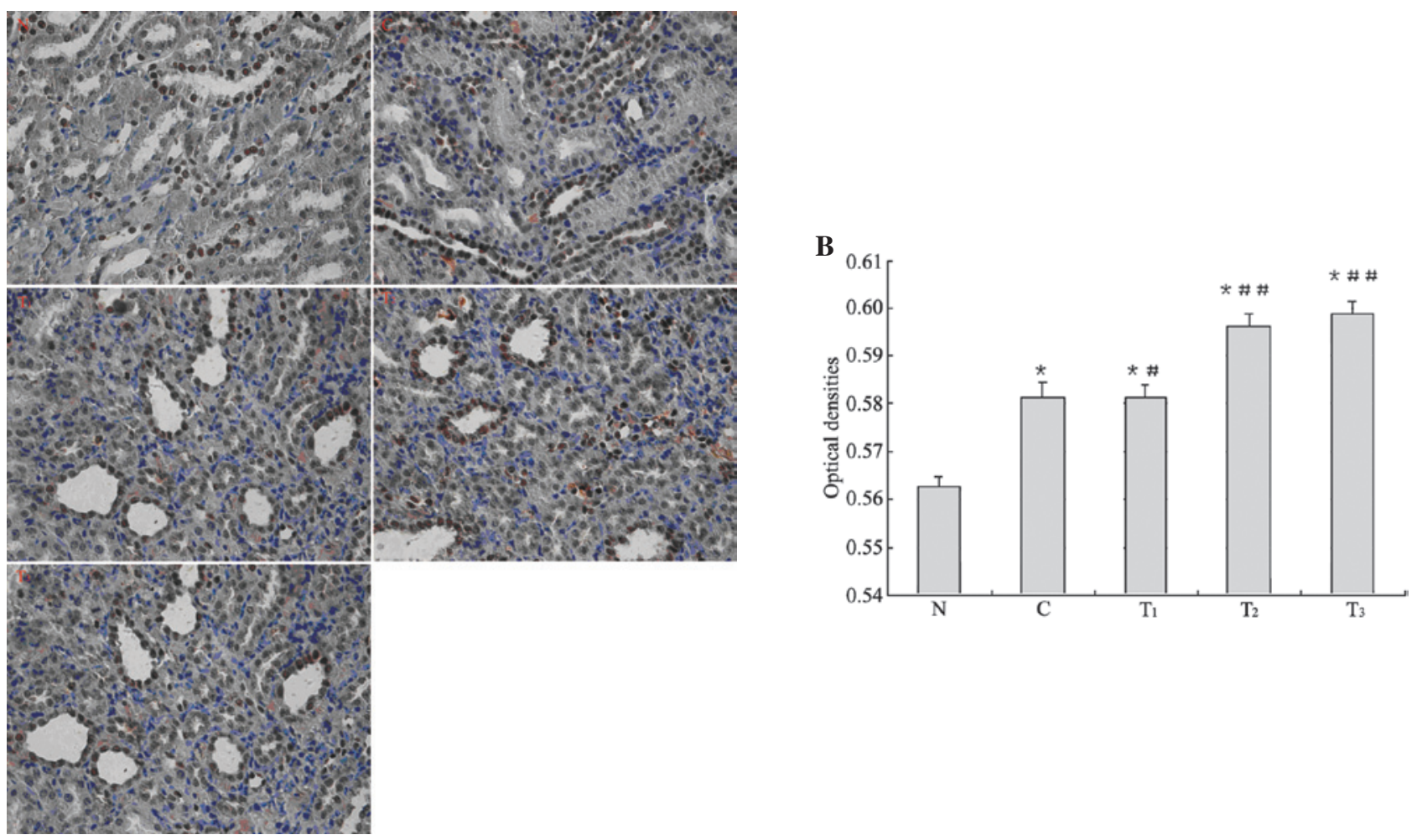

Figure 2. (A) Nrf2 expression and the effects of L-EGCG at different concentrations on (B) $\gamma$-GCS protein expression in different groups were analyzed using immunohistochemistry (x400). (A) Nrf2 expression is primarily located in the nuclei of renal tubular epithelial cells in all groups and is observed as puce-stained granules. (B) compared with group $\mathrm{N}$, both group $\mathrm{C}$ and the treatment groups display a significantly increased average optical density value $(\mathrm{P}<0.01)$. Compared with group $C$, group $T_{1}$ displays an increased average optical density value $(P<0.05)$ and groups $T_{2}$ and $T_{3}$ display significantly increased values $(P<0.01)$. N, normal control; C, model; $\mathrm{T}_{1}$, treatment with L-EGCG at $2.5 \mathrm{mg} / \mathrm{kg} ; \mathrm{T}_{2}$, treatment with L-EGCG at $5 \mathrm{mg} / \mathrm{kg} ; \mathrm{T}_{3}$, treatment with L-EGCG at $10 \mathrm{mg} / \mathrm{kg}$. ${ }^{*} \mathrm{P}<0.05$ and ${ }^{* *} \mathrm{P}<0.01$ compared with group $\mathrm{N} .{ }^{*} \mathrm{P}<0.05$ and ${ }^{\# \#} \mathrm{P}<0.01$ compared with group $\mathrm{C}$.

A
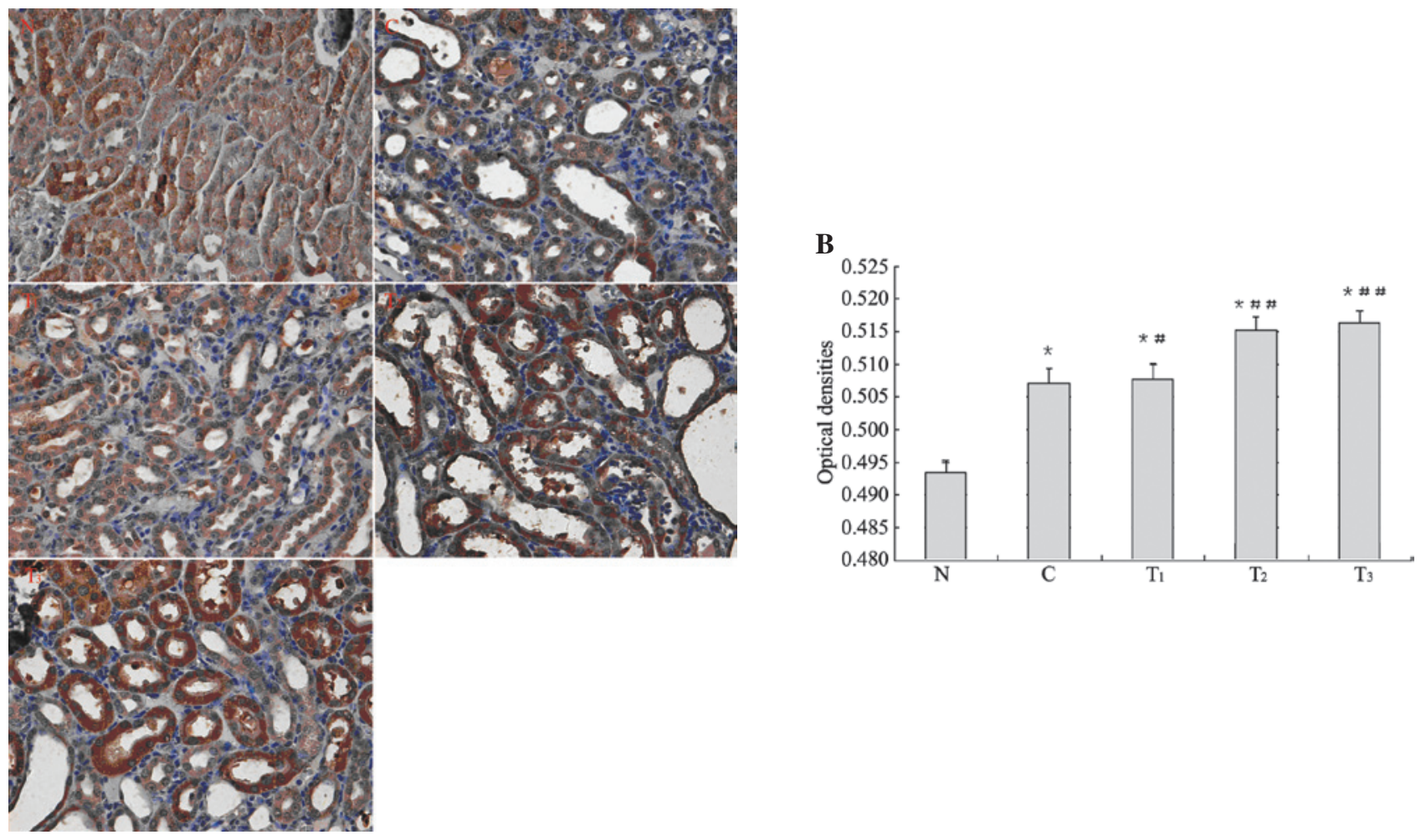

Figure 3. (A) $\gamma$-GCS expression and the dynamic effects of L-EGCG at different concentrations on the relative expression amounts of (B) Nrf2 mRNA in different groups were analyzed using immunohistochemistry (x400). (A) $\gamma$-GCS protein is primarily expressed in the cytoplasm of the renal tubular epithelial cells in all groups and observed as buffy- or puce-stained granules. (B) Compared with group $\mathrm{N}$, group $\mathrm{C}$ and the treatment groups display a significantly increased average optical density value $(P<0.01)$; compared with group $C$, group $T_{1}$ displays an increased average optical density value $(P<0.05)$ and groups $T_{2}$ and $T_{3}$ display significantly increased values $(\mathrm{P}<0.01)$. N, normal control; $\mathrm{C}$, model; $\mathrm{T}_{1}$, treatment with $\mathrm{L}-\mathrm{EGCG}$ at $2.5 \mathrm{mg} / \mathrm{kg} ; \mathrm{T}_{2}$, treatment with L-EGCG at $5 \mathrm{mg} / \mathrm{kg}$; $\mathrm{T}_{3}$, treatment with $\mathrm{L}-\mathrm{EGCG}$ at $10 \mathrm{mg} / \mathrm{kg} .{ }^{*} \mathrm{P}<0.05$ and ${ }^{* *} \mathrm{P}<0.01$ compared with group $\mathrm{N} .{ }^{*} \mathrm{P}<0.05$ and ${ }^{\# \#} \mathrm{P}<0.01$ compared with group $\mathrm{C}$. 

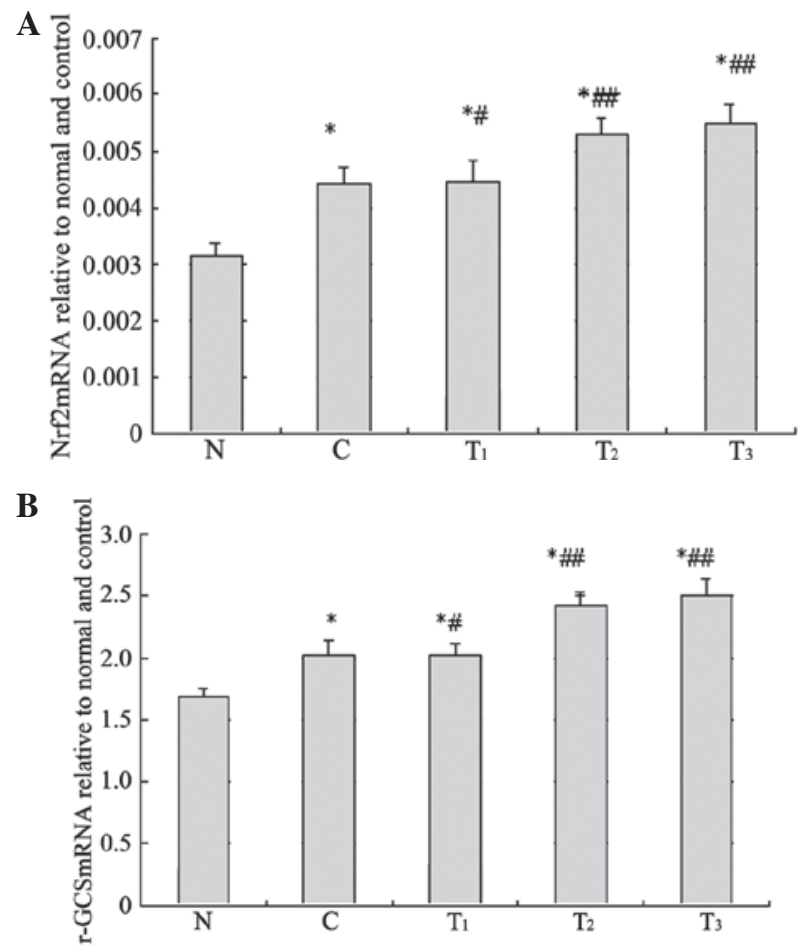

Figure 4. (A) Effect of different concentration of L-EGCG on Nrf2 mRNA expression of rat kidney tissues with acute obstructive nephropathy. (B) Effect of different concentration of L-EGCG on $\gamma$-GCS mRNA expression of rat kidney tissues with acute obstructive nephropathy. N, Normal control; $\mathrm{C}$, model; $\mathrm{T}_{1}$, treatment with $2.5 \mathrm{mg} / \mathrm{kg} \mathrm{L}-\mathrm{EGCG} ; \mathrm{T}_{2}$, treatment with $5 \mathrm{mg} / \mathrm{kg} \mathrm{L-EGCG} ; \mathrm{T}_{3}$, treatment with $2.5 \mathrm{mg} / \mathrm{kg} \mathrm{L-EGCG}$. Values represent the means \pm S.E.M. ${ }^{~} \mathrm{P}<0.05$ versus normal control group; ${ }^{*} \mathrm{P}<0.05,{ }^{\# \#} \mathrm{P}<0.01$ versus the model group.

Nrf2 and $\gamma$-GCS protein expression compared to the normal value $(\mathrm{p}<0.01)$, and the rats administered $2.5,5$ and $10 \mathrm{mg}$ of EGCG showed higher values compared to the control value, respectively. The levels of Nrf2 and $\gamma$-GCS in the kidney cortex of obstructive nephropathy control rats were similarly elevated above those in the kidney cortex of normal animals ( $<<0.01$, respectively), and treatment with EGCG at $2.5 \mathrm{mg}$ $(\mathrm{p}<0.05), 5$ and $10 \mathrm{mg}(\mathrm{p}<0.01)$ doses significantly enhanced the obstructive nephropathy-induced increases, respectively.

Electron microscopy. The ultrastructure of kidneys in rats from each group showed that the renal tissue was injured markedly in the model group (C), and the apoptosis of renal tubular epithelial cells induced by obstruction was alleviated in EGCG-treated groups $\left(\mathrm{T}_{2}\right.$ and $\left.\mathrm{T}_{3}\right)$ (Fig. 6).

\section{Discussion}

As an antioxidant, EGCG has drawn widespread attention in studies of kidney disease. EGCG has been known as the most potent Nrf2 activator among the green tea polyphenols, as evidenced by its pronounced ability to induce ARE-luciferase reporter gene transactivation (11). Nrf2, a bZIP transcription factor, is sequestered in the cytoplasm by kelch-like ECH-associated protein 1 (Keap1). Exposure of cells to the antioxidant response element (ARE) inducers results in the dissociation of Nrf2 from Keap1 and facilitates translocation of Nrf2 to the nucleus, where it heterodimerizes with
$\mathbf{A}$
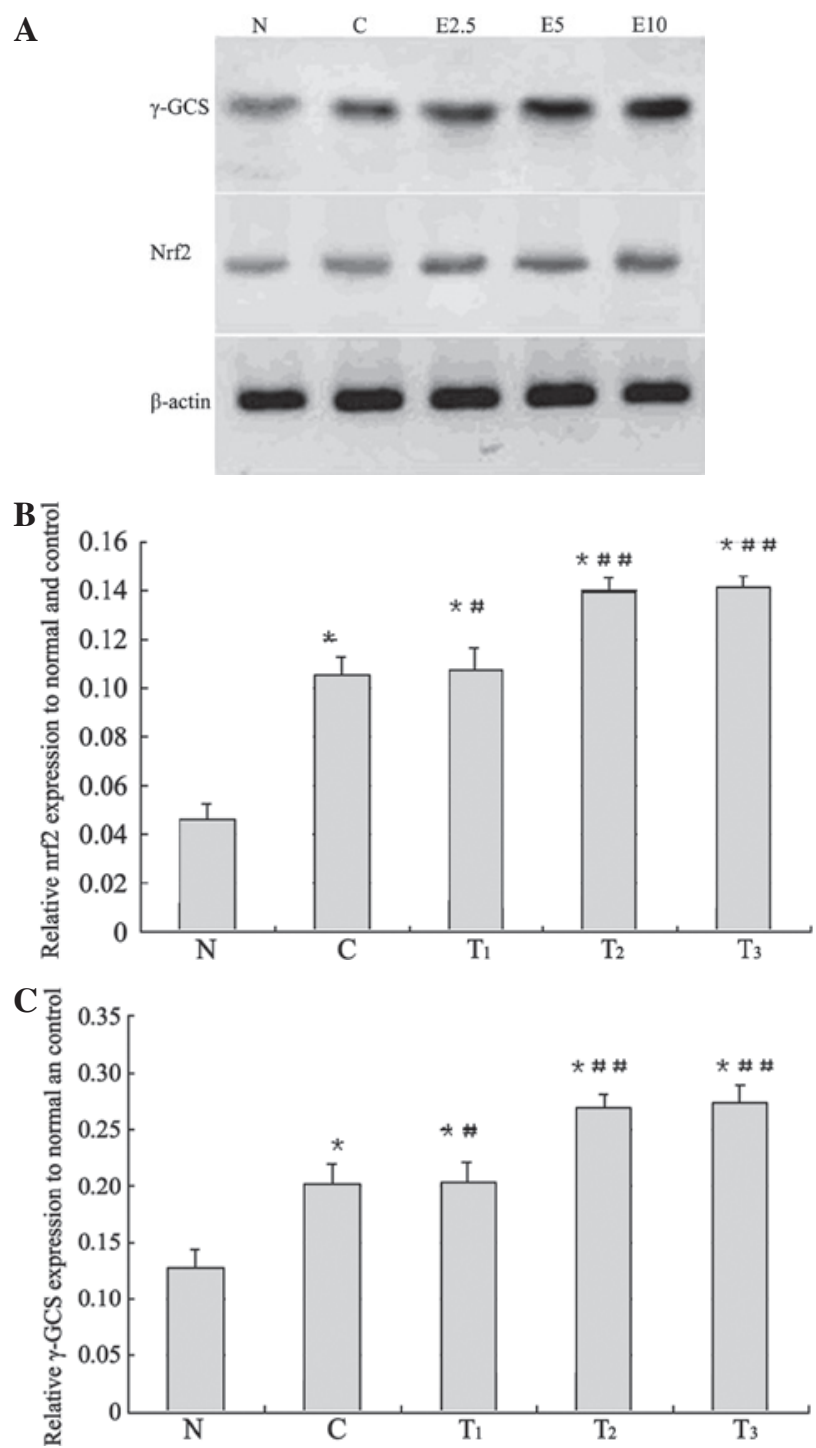

Figure 5. (A) The expression of Nrf2 and $\gamma$-GCS protein in the renal cortex and medulla of the different groups was detected using western blot analysis. (B) Compared with group $\mathrm{N}$, group $\mathrm{C}$ displays a significant increase in Nrf2 protein expression $(\mathrm{P}<0.01)$; compared with group $\mathrm{C}$, group $\mathrm{T}_{1}$ shows an increase in Nrf2 protein expression $(\mathrm{P}<0.05)$ and both $\mathrm{T}_{2}$ and $\mathrm{T}_{3}$ show a significant increase $(\mathrm{P}<0.01)$. (C) Compared with group $\mathrm{N}$, group $\mathrm{C}$ displays a significant increase in $\gamma$-GCS protein expression $(\mathrm{P}<0.01)$; compared with group $C$, group $T_{1}$ shows an increase in $\gamma$-GCS protein expression $(\mathrm{P}<0.05)$ and both $\mathrm{T}_{2}$ and $\mathrm{T}_{3}$ show a significant increase $(\mathrm{P}<0.01)$. N, normal control; $\mathrm{C}$, model; $\mathrm{T}_{1}$, treatment with L-EGCG at $2.5 \mathrm{mg} / \mathrm{kg} ; \mathrm{T}_{2}$, treatment with L-EGCG at $5 \mathrm{mg} / \mathrm{kg} ; \mathrm{T}_{3}$, treatment with L-EGCG at $10 \mathrm{mg} / \mathrm{kg}$. ${ }^{*} \mathrm{P}<0.05$ and ${ }^{* *} \mathrm{P}<0.01$ compared with group $\mathrm{N}$. ${ }^{\#} \mathrm{P}<0.05$ and ${ }^{\# \#} \mathrm{P}<0.01$ compared with group $\mathrm{C}$.

small Maf protein, and binds to ARE, eventually resulting in the transcriptional regulation of target genes including both phase I (oxidation and reduction) and phase II biotransformation (conjugation) (12-15). The molecular mechanism underlying antioxidant enzyme induction by EGCG has been the subject of extensive investigations. One of the most plausible mechanisms responsible for activation of Nrf2 involves phosphorylation of serine/threonine residues of Nrf2 by protein kinases, which facilitates enhanced nuclear translocation of Nrf2 and subsequent ARE binding (16). The phenomenon of nuclear translocation was also observed by Nrf2 immunohistochemical staining in this study, that is, 


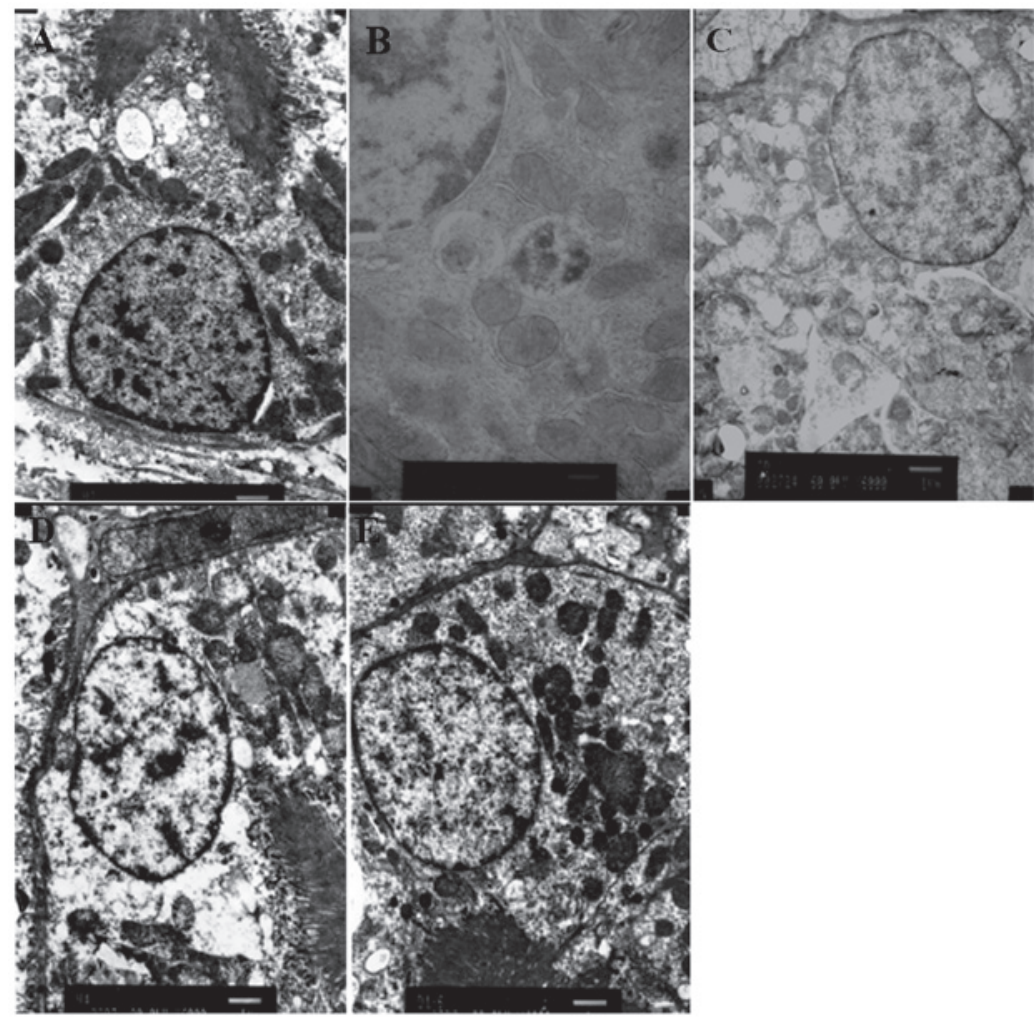

Figure 6. Electron microscope from the kidney cortex and medulla of experimental animals. (A) Group N: intact proximal renal tubular epithelial cell and microvilli were observed (arrow). (B) Group C: the englobed apoptotic bodies in the tubular epithelial cells were observed (arrow). (C) L-EGCG group ( $2.5 \mathrm{mg} / \mathrm{kg} /$ day): nuclear irregular and mitochondrial vacuolar degeneration accompanied by reduced crest and missing surface microvilli were observed. The basement membrane was continuous. (D) L-EGCG group $(5 \mathrm{mg} / \mathrm{kg} / \mathrm{day})$ : proximal tubule basement membrane integrity was seen and the microvilli were intact, and there were dissolved mitochondria ridges (arrow). (E) L-EGCG group (10mg/kg/day) : proximal renal tubular pile and tubular basement membrane were intact and clear, rough endoplasmic reticulum was observed.

Nrf2-positive cells transferred from the cytoplasm to the nuclei of renal tubular epithelial cells. These findings of EGCG upregulation and nuclear translocation on Nrf2 correspond with other studies (16-18). EGCG has been shown to induce expression of glutathione S-transferase, glutathione peroxidase, $\gamma$-GCS and heme oxygenase-1, which are involved in the elimination or inactivation of ROS and electrophiles implicated in multi-stage oxidative stress. $\gamma$-GCS may be considered to be one of the major antioxidant enzymes, as it is the rate-limiting enzyme in GSH synthesis and GSH has been postulated to be one of the most important antioxidants $(19,20)$. Nrf2 gene knockout of rats may lead to the reduction of $\gamma$-GCS subunit expression and GSH synthesis in fibroblast cells and liver cells (21). These results thus provide new insights into the anti-oxidative mechanisms of EGCG. These findings suggest that EGCG-induced expression of some representative antioxidant enzymes may provide the rats with acquired antioxidant defense capacity, allowing them to survive oxidative stress.

The role of this study was to ascertain the possible roles of Nrf2 in renal cellular defense against oxidative stress, and whether EGCG could ameliorate the development of obstructive nephropathy. The data indicated that oxidant stress could improve the protein expression of Nrf2 and $\gamma$-GCS, and the expression could be enhanced by EGCG. The protein expression of both Nrf2 and $\gamma$-GCS is mainly located in renal tubular epithelial cells. It is thus clear that oxidative stress of rats with
UUO mainly injured the renal tubule, which suggests that renal oxidative stress may increase the downstream target gene $\gamma$-GCS through upregulation of Nrf2.

Nrf2 plays a pivotal role in cell defenses against oxidative stress in the kidney by controlling the intracellular antioxidant states, and the possibility of Nrf2 participation in prevention of systemic oxidative stress is proposed in this study. In addition, EGCG-treated groups showed suppressed oxidative stress and acute renal damage caused by UUO. Our study demonstrates that UUO rats were much more susceptible to the expression of Nrf2 and $\gamma$-GCS, and EGCG induces Nrf2-mediated expression of $\gamma$-GCS in renal tissue. Therefore, we inferred that EGCG activated Keap1, facilitated the release of Nrf2 for nuclear translocation, and finally induced expression of some representative antioxidant enzymes in renal tissue.

The expression of Nrf2 and $\gamma$-GCS were positively correlated with the doses of EGCG administered by intraperitoneal injection, and high-dose EGCG had the best oxidative stress protective effect. Results from this study also provide important and novel insights into the molecular mechanisms underlying the oxidative stress chemoprevention effects of EGCG, as well as the role of Nrf2 in its biological functions. EGCG showed marked anti-oxidative activity in the management of obstructive nephropathy in a dose-dependent manner. Our results indicate that the development of obstructive nephropathy may be effectively inhibited by EGCG. As 
one of the most powerful antioxidants with the advantages of high safety and fewer adverse reactions, EGCG is expected to be an effective application for the prevention or treatment of obstructive nephropathy. Of course, these conclusions are only based on animal experiments, and further studies are required to establish this type of treatment in humans. A larger number of clinical experiments must be performed to verify the chemopreventive effects of EGCG, in order for EGCG to have future clinical application.

\section{Acknowledgements}

This study was supported by grants of Liaoning Province Natural Science Foundation of China and the Science and Technology Research Foundation, Department of Education of Heilong jiang Provincial (11551158). The authors thank the Key Laboratory of Congenital Malformations of China Ministry of Health for providing the experiment site and instruments. The authors also wish to express their gratitude to Li Ma for her technical assistance and Dr Jia-ning Miao for his assistance with histopathological-related research.

\section{References}

1. Klahr S: Obstructive Nephropathy. Internal Med 39: 355-361, 2000.

2. Robertson JL: Chemically induced glomerular injury. A review of basic mechanisms and specific xenobiotics. Toxicol Pathol 26 64-72, 1998.

3. Zalba G, Fortuño A and Díez J: Oxidative stress and atherosclerosis in early chronic kidney disease. Nephrol Dial Transplant 21: 2686-2690, 2006.

4. Yang CS: Inhibition of carcinogenesis by tea. Nature 389: 134-135, 1997.

5. Fujiki H, Suganuma M, Okabe S, et al: Cancer inhibition by green tea. Mutat Res 402: 307-310, 1998.

6. Mukhtar H and Ahmad N: Mechanism of cancer chemopreventive activity of green tea. Proc Soc Exp Biol Med 220 234-238, 1999.

7. Suganuma M, Okabe S, Sueoka N, et al: Green tea and cancer chemoprevention. Mutat Res 428: 339-344, 1999.

8. Robak J and Gryglewski RJ: Flavonoids are scavengers of superoxide anions. Biochem Pharmacol 37: 837-841, 1988.
9. Yang CS, Lambert JD, Hou Z, Ju J, Lu G and Hao X: Molecular targets for the cancer preventive activity of tea polyphenols. Mol Carcinog 45: 431-435, 2006.

10. Yang CS, Prabhu S and Landau J: Prevention of carcinogenesis by tea polyphenols. Drug Metabolism Reviews 33: 237-253, 2001.

11. Chen C, Yu R, Owuor ED and Kong AN: Activation of antioxidant-response element (ARE), mitogen-activated protein kinases (MAPKs) and caspases by major green tea polyphenol components during cell survival and death. Arch Pharm Res 23: 605-612, 2000.

12. Cho HY, Jedlicka AE, Reddy SP, et al: Role of NRF2 in protection against hyperoxic lung injury in mice. Am J Respir Cell Mol Biol 26: 175-182, 2002.

13. Thimmulappa RK, Mai KH, Srisuma S, Kensler TW, Yamamoto $\mathrm{M}$ and Biswal S: Identification of Nrf2-regulated genes induced by the chemopreventive agent sulforaphane by oligonucleotide microarray. Cancer Res 62: 5196-5203, 2002.

14. Hu R, Xu C, Shen G, et al: Identification of Nrf2-regulated genes induced by chemopreventive isothiocyanate PEITC by oligonucleotide microarray. Life Sci 79: 1944-1955, 2006.

15. Shelby MK and Klaassen CD: Induction of rat UDP-glucuronosyltransferases in liver and duodenum by microsomal enzyme inducers that activate various transcriptional pathways. Drug Metab Dispos 34: 1772-1778, 2006.

16. Wu CC, Hsu MC, Hsieh CW, Lin JB, Lai PH and Wung BS: Upregulation of heme oxygenase-1 by Epigallocatechin-3-gallate via the phosphatidylinositol 3-kinase/Akt and ERK pathways. Life Sci 78: 2889-2897, 2006.

17. Yang XY, Zhao WP, Li YQ, et al: The role of NF-E2-related factor 2 in the induction of uridine 5-diphosphate-glucuronosyltransferase 1A and its isoforms by epigallocatechin gallate in colon cancer cells. Zhonghua Yi Xue Za Zhi 86: 82-87, 2006.

18. Andreadi CK, Howells LM, Atherfold PA and Manson MM: Involvement of Nrf2, p38, BRaf, and nuclear factor-kappaB, but not phosphatidylinositol 3-kinase, in induction of hemeoxygenase-1 by dietary polyphenols. Mol Pharmacol 69: 1033-1040, 2006.

19. Dalton TP, Dieter MZ, Yang Y, Shertzer HG and Nebert DW: The most glutamate cysteine ligase catalytic subunit (Gclc) gene: embryonic lethal when homozygous, and proposed model for moderate glutathione deficiency when heterzygous. Biochem Biophys Res Commun 20: 279, 2000.

20. Itoh K, Chiba T, Takahashi S, et al: An Nrf2/small Maf heterodimer mediates the induction of phase II detoxifying enzyme genes through antioxidant response elements. Biochem Biophys Res Commun 236: 313-322, 1997.

21. Chan JY and Kwong M: Impaired expression of glutathione synthetic enzyme genes in mice with targeteddeletion of the Nrf2 basic-leucine zipper protein. Biochim Biophys Acta 1517: 19-26, 2000 . 